\title{
BMJ Open Relationship between initial therapy and blood pressure control for high-risk hypertension patients in the UK: a retrospective cohort study from the THIN general practice database
}

\author{
Sharada Weir, ${ }^{1,2}$ Attila Juhasz, ${ }^{3}$ Jorge Puelles, ${ }^{4}$ Travis S Tierney ${ }^{5}$
}

To cite: Weir S, Juhasz A, Puelles J, et al. Relationship between initial therapy and blood pressure control for highrisk hypertension patients in the UK: a retrospective cohort study from the THIN general practice database. BMJ Open 2017;7:e015527. doi:10.1136/ bmjopen-2016-015527

- Prepublication history and additional material are available. To view these files, please visit the journal online (http://dx.doi. org/10.1136/bmjopen-2016015527).

Received 13 December 2016 Revised 7 June 2017

Accepted 21 June 2017

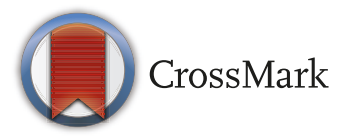

${ }^{1}$ Centre for Addiction and Mental Health, University of Toronto, Toronto, Canada

${ }^{2}$ PHMR Limited, London, UK ${ }^{3}$ Department of Clinical

Sciences, Takeda Development Centre Europe Limited, London, UK

${ }^{4}$ Takeda Development Centre Europe Limited, Global Outcomes Research, London, UK ${ }^{5}$ Department of Medicine, Imperial College London, UK

Correspondence to

Dr Sharada Weir;

sharadaweir@phmr.com

\section{ABSTRACT}

Objective To examine the UK practice patterns in treating newly diagnosed hypertension and to determine whether subgroups of high-risk patients are more or less likely to follow particular therapeutic protocols and to reach blood pressure goals.

Design Retrospective cohort study.

Setting This study examined adults in The Health Improvement Network (THIN) UK general practice medical records database who were initiated on medication for hypertension.

Participants 48131 patients with essential hypertension diagnosed between 2008 and 2010 who were registered with a participating practice for a minimum of 13 months prior to, and 6 months following, initiation of therapy. We excluded patients with gestational hypertension or secondary hypertension. Patients were classified into risk groups based on blood pressure readings and comorbid conditions.

Primary and secondary outcome measures 0dds of receiving single versus fixed or free-drug combination therapy and odds of achieving blood pressure control were assessed using multivariable logistic regression. Results The vast majority of patients (95.8\%) were initiated on single drug therapy. Patients with high cardiovascular risk (patients with grade 2-3 hypertension or those with high normal/grade 1 hypertension plus at least one cardiovascular condition pretreatment) had a statistically significant benefit of starting immediately on combination therapy when blood pressure control was the desired goal (OR: 1.23; $95 \% \mathrm{Cl}: 1.06$ to 1.42 ) but, surprisingly, were less likely than patients with no risk factors to receive combination therapy (OR: $0.53 ; 95 \% \mathrm{Cl}: 0.47$ to 0.59 ).

Conclusions Our results suggest that combination therapy may be indicated for patients with high cardiovascular risk, who accounted for $60.6 \%$ of our study population. The National Institute for Health and Care Excellence guideline CG34 of 2006 (in effect during the study period) recommended starting with single drug class therapy for most patients, and this advice does seem to have been followed even in cases where a more aggressive approach might have been considered.
Strengths and limitations of this study

- This is one of the largest nationally representative studies of hypertension practice and outcomes in the UK.

- We had access to a very large general practice dataset to identify patient risk factors, but without data on inpatient encounters, the proportion of highrisk patients may have been underestimated.

- The dataset benefited from near complete reporting of follow-up blood pressure readings after therapy initiation, but the 6-month follow-up period precluded analysis of long-term blood pressure control outcomes.

- It may be beneficial to extend this analysis using data from 2012 onwards to assess the impact of the updated 2011 National Institute for Health and Care Excellence guidelines on choice of therapeutic agents among particular subgroups of the population and whether these choices affected outcomes in clinical practice.

\section{INTRODUCTION}

Hypertension-generally defined by sustained blood pressure $(\mathrm{BP}) \geq 140 / 90 \mathrm{~mm}$ $\mathrm{Hg}$-is one of the most common premorbid conditions contributing to deadly disease in the UK. The Health Survey for England reported the prevalence of hypertension to be $27.9 \%$ in those aged $40-79$ years rising to $49.9 \%$ in those aged over 80 years. A similarly high prevalence of hypertension is seen in adults in nearly every country throughout the high-income world. ${ }^{12}$

More than $7 \%$ of deaths worldwide are directly attributable to hypertension, exceeding rates for tobacco use and high cholesterol. ${ }^{3}$ Hypertension has been estimated to confer a $3 \%-19 \%$ increase in the risk of stroke and a $3 \%$ increase in the risk of developing heart failure. It may account for $25 \%$ of deaths from coronary artery 
disease. $^{2}$ Patients with hypertension and comorbid diabetes, obesity or hyperlipidaemia have been found to be at even higher risk for cardiovascular disease and end-organ damage. ${ }^{4}$ Hypertension places an extraordinarily high economic burden on healthcare systems through the high-income world. ${ }^{25}$

At the same time, hypertension is one of the most significant, single, modifiable risk factors associated with cardiovascular disease and stroke, and appropriate treatment has been shown to significantly reduce both morbidity and mortality associated with these conditions. ${ }^{267}$ Together with diet and lifestyle modifications, a range of pharmaceutical therapies have been found to be highly effective in controlling hypertension. ${ }^{8}$

Recommended initial therapy for patients with hypertension varies from country to country. In the UK, physicians are advised to start patients on monotherapy and add an additional drug only in the case of failure to reach BP goal on an adequate dose of a single drug. ${ }^{9} 10$ The European guidelines have for more than a decade emphasised the importance of considering additional co-occurring cardiovascular, renal and metabolic conditions when initiating treatment for hypertension, recommending different strategies depending on overall cardiovascular risk. ${ }^{11}$

The purpose of this study is to examine real-world practice in the treatment of newly diagnosed hypertension in the UK, comparing treatment pathways for low-risk and high-risk individuals. Our aim is to determine whether particular subgroups of patients (eg, those with diabetes, renal disease or additional cardiovascular risk factors) are more or less likely than others to follow particular therapeutic protocols and to meet immediate BP goals following therapy initiation.

\section{METHODS}

To investigate initial therapy for new onset hypertension in the UK, we acquired patient-level data from The Health Improvement Network ('THIN'), a computerised database of anonymised longitudinal medical records covering approximately 500 UK primary care practices, over a 3-year period, from 2008 to 2010.

The THIN database covers $5.7 \%$ of the UK population $^{12}$ and captures patient demographics and practice enrolment dates, diagnoses, referrals to secondary care, prescriptions, laboratory results and measurements taken during patient visits. ${ }^{13} 14$ These data have been used to study patients with hypertension in the past. ${ }^{15-17}$

Approval of the THIN Scheme was granted by the NHS South-East Multicentre Research Ethics Committee (MREC) in 2002. ${ }^{18}$ Per requirements of the MREC, the present study was granted scientific approval by the data vendor's Scientific Review Committee in March 2012. The study protocol is available as a web supplement to this article. This manuscript was prepared in compliance with the STrengthening the Reporting of OBservational studies in Epidemiology (STROBE) guidelines for cohort studies (checklist included as a web supplement) ${ }^{19}$

\section{Study design}

We conducted a retrospective cohort study of adults newly treated for hypertension during calendar years 2008-2010. Patients were required to be continuously registered at a practice for a minimum of 19 months during this period.

The study population included adults (ages 18 and older) with newly treated primary (essential) hypertension as identified by a Read diagnosis code in the electronic medical record (EMR) indicating essential hypertension. Patients with gestational hypertension and secondary hypertension were excluded.

We used diagnosis codes, rather than use of actual BP readings, to define hypertension since that approach better allows for exclusion of secondary and gestational hypertension as well as hypertensive emergencies and other causes of high BP not associated with primary hypertension.

To identify newly treated hypertension, we imposed a preindex 'clean' period of a minimum of 13 months during which patients did not receive a prescription for an antihypertensive medication. This period was chosen since well-controlled patients with hypertension may be expected to visit their general practitioner (GP) at least annually for follow-up. We allowed an extra month in case of delay in scheduling an annual appointment to obtain a prescription renewal.

The index date was the date of the first prescription for an antihypertensive medication following at least 13 months free from such medication at the outset of the study period.

Patients were followed for a period of 6 months after index treatment initiation (post-treatment period) to allow time to observe the effects of treatment on hypertension outcomes.

\section{Exposures, outcomes and covariates}

Antihypertensive therapy. Hypertension guidelines recognise five primary drug classes: thiazide/thiazide-like diuretics, beta-blockers, calcium channel blockers (CCBs), ACE inhibitors (ACEIs) and angiotensin receptor blockers (ARBs). This study examined the use of the five primary classes of antihypertensive medications, as monotherapy or in combination, as well as other antihypertensive drugs used in general practice. Relevant drugs were identified using codes from Chapter 2 of the British National Formulary.

BP control outcomes. Systolic and diastolic BP readings were obtained from the EMR. The last recorded measurement during the periods immediately prior to treatment initiation (pretreatment period) and in the 6 months following treatment initiation (post-treatment period) were used to categorise patients into hypertension grade, preindex and postindex. A patient was classified into the highest grade appropriate based on either their systolic or diastolic reading. BP was defined as 'in control' or 
'out of control' in the post-treatment period depending on BP readings in relation to the threshold target recommended by the National Institute for Health and Care Excellence (NICE) of 140/90.

Covariates. Independent variables were constructed based on the index date (patient demographics and socioeconomic status) or preindex period (lifestyle characteristics and chronic/comorbid conditions).

i) Patient demographics: age (in years) and sex; ii) patient lifestyle variables (measured using Read codes recorded during the preindex study period): tobacco use (defined as current smoker) and overweight/ obese status (measured as BMI $\geq 30$ or Read code indicating overweight/obese) and iii) chronic conditions (measured using Read codes for all diagnoses on record up to the index treatment date): diabetes mellitus, renal disease, coronary heart disease (not including myocardial infarction), cerebrovascular disease, peripheral vascular disease, myocardial infarction or hyperlipidaemia.

\section{Risk cohorts}

Patients were assigned to risk groups based on a combination of their pretreatment $\mathrm{BP}$ grade and the presence of comorbid conditions following criteria outlined by Mancia $e t a l^{11}$ in their guidelines for management of hypertension. ${ }^{11}$ A patient was considered 'high risk' on the basis of potential cardiovascular morbidity if he or she had a pretreatment hypertension grade of 2 or 3 or if $\mathrm{BP}$ was in the high-normal to mild range in the presence of one or more key cardiovascular conditions (ie, coronary heart disease, cerebrovascular disease, peripheral vascular disease, myocardial infarction or hyperlipidaemia). Patients with kidney disease (with or without diabetes) and those with diabetes (without coexisting kidney disease) were also considered 'high risk'. All others were classified as 'low risk'.

\section{Missing data}

The UK medical records typically provide nearly complete data for the key study variables identified here. The UK 'Quality and Outcomes Framework', ${ }^{20}$ introduced in 2004, provides financial incentives for the UK GPs to appropriately document important metrics and meet selected quality process and outcome goals. Physicians are paid incentive bonuses for keeping a registry of patients with hypertension being treated in their practice and for recording BP in hypertensive patients every 9 months, at a minimum. For patients with diabetes or kidney disease, additional incentives are offered to regularly monitor $\mathrm{BP}$ regardless of whether hypertension has been diagnosed. Incentives are also provided for keeping a registry of patients with BMI $\geq 30$ in the prior 15 months and for recording smoking status among patients with hypertension or other cardiovascular or metabolic conditions.

For the purposes of analyses, continuous variables (such as BMI) were recoded into categories. Where data were missing, the patient was assumed to fall into the reference category. The exception was BP recordings, where we created a separate category for missing data.

\section{Statistical analysis}

We employed a mix of descriptive analyses and logistic regression analyses using SAS software, V.9.3 for Windows.

Simple descriptive statistics were included to illustrate characteristics of the population, initial treatment regimen, and $\mathrm{BP}$ control status in the 13-month pretreatment period and in the 6-month post-treatment period.

Logistic regression models were used to examine the association between patient characteristics and outcomes of interest. Risk groups were identified in the models and separate models were run for each risk group to examine interactions. Analyses were restricted to patients who had follow-up BP recorded as this was necessary to evaluate the outcome of $\mathrm{BP}$ control. All models were subjected to the Hosmer-Lemeshow goodness-of-fit test. Since this test may be sensitive to sample size ${ }^{21}$ we also calculated the c-statistic. Effects are expressed as ORs. Statistical significance of independent variables in each model was evaluated at the $\alpha<0.05$ level. Bonferroni correction was used to maintain this family-wise error rate in the presence of multiple pair-wise comparisons.

A sensitivity analysis was performed to assess the potential impact of missing data on BP readings. The BP control regression analysis was rerun twice for each risk group and for all patients: under the first scenario, we assumed that all patients with missing BP readings had achieved BP control following treatment; under the second scenario, we assumed that they had not.

\section{RESULTS}

\section{Study population}

A total of 48131 patients were found to meet all study criteria. Just over half of the population was male with a mean age of 57.3 years. Table 1 summarises demographic and key lifestyle variables by risk group. One-third of patients had been diagnosed with one or more risk-elevating comorbid conditions (diabetes, renal disease and cardiovascular disease) prior to index treatment initiation. Others were classified as high risk based on pretreatment BP readings indicating grade 2 or 3 hypertension. We found high rates of overweight/obesity and smoking across groups.

\section{Index antihypertensive therapy}

The vast majority of patients $(95.8 \%)$ were initiated on single drug therapy. Table 2 shows the distribution of patients by index treatment pathway and risk cohort. Combination therapy (either fixed dose combination drugs or multiple single agents prescribed on the index date) was highest for patients with renal disease, at $6.0 \%$, and lowest for patients in the cardiovascular risk group (grade 2 or 3 hypertension pretreatment or those with high-normal or grade 1 hypertension in combination with one or more cardiovascular conditions). The most common drug class used in monotherapy, across all risk classes, was ACEIs, followed by CCBs. 
Table 1 Age and sex distribution of the study population (\%)

\begin{tabular}{|c|c|c|c|c|c|}
\hline & \multicolumn{3}{|c|}{ High-risk patients } & \multirow[b]{2}{*}{ Low-risk patients } & \multirow{2}{*}{$\begin{array}{l}\text { All } \\
\text { patients }\end{array}$} \\
\hline & Kidney disease & Diabetes mellitus & Cardiovascular* & & \\
\hline \multicolumn{6}{|l|}{ Age, years } \\
\hline Mean & 67.0 & 56.8 & 57.1 & 55.6 & 57.3 \\
\hline Median & 69.0 & 57.0 & 57.0 & 56.0 & 57.0 \\
\hline Male, \% & 42.0 & 58.2 & 50.9 & 50.5 & 50.9 \\
\hline Obese/overweight, \% & 61.4 & 83.8 & 66.4 & 65.7 & 67.5 \\
\hline Current tobacco use, \% & 20.3 & 25.8 & 25.1 & 23.7 & 24.5 \\
\hline Number of patients & 3060 & 4303 & 29175 & 11593 & 48131 \\
\hline$\%$ of patients & 6.4 & 8.9 & 60.6 & 24.1 & 100.0 \\
\hline
\end{tabular}

*The cardiovascular risk group includes patients with grades 2 or 3 hypertension (with or without comorbid cardiovascular disease) prior to treatment initiation and those with 'high normal' or grade 1 hypertension plus one or more cardiovascular conditions (ie, coronary heart disease, cerebrovascular disease, peripheral vascular disease, myocardial infarction or hyperlipidaemia).

Table 3 shows the results of a multivariable logistic regression of the odds of receiving combination therapy as a function of patient characteristics, including risk cohort. The first model included only patient characteristics (other than risk group), the second included risk groups alone, unadjusted for other patient characteristics and the third model included risk groups adjusted for patient characteristics (excluding pretreatment hypertension grade which was included in the definition of the cardiovascular risk group). In model 3, we excluded comorbid conditions since the presence of one or more of these conditions is an integral part of the definition of the high-risk groups that were included in this model.

Model 1 shows that men, patients who registered with the practice during the study period, those who had an episode of hypertension prior to the study period and current smokers all had higher odds of receiving initial treatment with combination therapy. Odds of receiving combination therapy were lower, other things equal, among those with higher grade hypertension in the immediate pretreatment period. However, the association was non-linear. Patients with grade 2 hypertension had the lowest odds of receiving combination therapy. Patients who did not have a BP reading recorded for the pretreatment period (3.7\% of all patients) were not significantly less likely to receive combination therapy than those who did.

Model 2 shows that patients with diabetes (OR: 0.64; 95\% CI: 0.53 to 0.77 ) and cardiovascular disease (OR: 0.52 ; $95 \%$ CI: 0.47 to 0.58 ) were both less likely than those with no risk factors to receive combination therapy. Adjusting for demographics and lifestyle factors in model 3 did not alter our findings.

\section{BP control}

More than two-thirds of patients $(66.8 \%)$ had grade 2 hypertension or higher prior to treatment, falling to $13.5 \%$ in the postindex period. Low-risk patients were, by definition, those who had no worse than grade 1 hypertension prior to treatment and without risk-elevating comorbid conditions (diabetes, renal disease and cardiovascular disease). A shift from grade 2 or 3 hypertension towards grade 1 (or lower) was observed in the follow-up period in all other subjects, including those with cardiovascular conditions $(96.4 \%$ were classified in grades 2 or 3 pretreatment vs $16.3 \%$ afterwards), diabetes $(53.3 \%$ vs $11.5 \%)$ and kidney disease (56.1\% vs $10.9 \%)$. Among low-risk patients, results were somewhat less dramatic

Table 2 Monotherapy vs fixed-drug or free-drug combination therapy, by risk cohort, $n=48131$ (\%)

\begin{tabular}{lccccc}
\hline Antihypertensive drug class & Kidney disease & Diabetes mellitus & $\begin{array}{l}\text { High-risk } \\
\text { cardiovascular* }\end{array}$ & Low-risk patients & All patients \\
\hline Combination therapy & 6.0 & 4.0 & 3.2 & 6.3 & 4.2 \\
\hline Monotherapy & & & & \\
\multicolumn{1}{c}{ ACE inhibitors } & 40.3 & 61.5 & 43.0 & 36.6 & 42.3 \\
\hline Angiotensin receptor blockers & 3.4 & 3.5 & 2.2 & 2.5 & 2.4 \\
\hline Calcium channel blockers & 25.4 & 16.7 & 30.8 & 22.6 & 27.7 \\
\hline Diuretics & 17.2 & 9.1 & 15.4 & 17.8 & 15.7 \\
\hline Beta-blockers & 4.9 & 3.3 & 4.4 & 10.3 & 5.9 \\
\hline Other antihypertensive drugs & 2.8 & 1.8 & 1.1 & 3.9 & 1.9 \\
\hline
\end{tabular}

Columns may not sum to $100 \%$ because of rounding. 
Table 3 Odds of receiving fixed-drug or free-drug combination therapy vs monotherapy as index treatment

\section{Model 1: \\ Model 2: \\ Model 3:}

patient variables only

Variable OR $(95 \% \mathrm{Cl})$

risk groups only OR $(95 \% \mathrm{Cl})$

Adjusted

OR $(95 \% \mathrm{Cl})$

\section{Age cohort}

Age $<55$

Age $\geq 55$

0.946 (0.848 to 1.055$)$

1.114 (1.004 to 1.236$)$

Sex

\section{Female}

Male

1.385 (1.248 to 1.537$)$

1.552 (1.404 to 1.716$)$

Registration with practice

Existing patient

New patient

1.661 (1.301 to 2.120$)$

1.715 (1.353 to 2.174$)$

History of hypertension

No prior hypertension

Prior episode of hypertension

1.756 (1.580 to 1.952$)$

2.144 (1.938 to 2.371$)$

Lifestyle factors

Not current smoker

Current smoker

1.245 (1.113 to 1.394$)$

1.269 (1.138 to 1.415$)$

Not obese/overweight

Overweight $\quad 0.972$ (0.858 to 1.102)

Obese

1.073 (0.945 to 1.218$)$

0.904 (0.801 to 1.020$)$

0.956 (0.846 to 1.081$)$

Comorbid conditions

\begin{tabular}{|c|c|}
\hline Diabetes & $0.812(0.680$ to 0.971$)$ \\
\hline Kidney disease & $1.123(0.932$ to 1.353$)$ \\
\hline Coronary heart disease & 2.980 (2.207 to 4.024$)$ \\
\hline Cerebrovascular disease & 1.692 (1.397 to 2.050$)$ \\
\hline Peripheral vascular disease & 0.976 (0.749 to 1.270$)$ \\
\hline Myocardial infarction & 5.252 (4.498 to 6.133$)$ \\
\hline Hyperlipidaemia & $0.916(0.799$ to 1.050$)$ \\
\hline
\end{tabular}

Pretreatment hypertension grade

Lower than grade 1

Grade $1 \quad 0.272(0.230$ to 0.322$)$

Grade 2

0.185 (0.157 to 0.218$)$

Grade 3

0.352 (0.299 to 0.415$)$

No pretreatment BP reading

0.857 (0.693 to 1.060$)$

Risk group

\begin{tabular}{|c|c|c|c|}
\hline \multicolumn{2}{|l|}{ Diabetes mellitus } & $0.639(0.530$ to 0.771$)$ & 0.597 (0.494 to 0.721$)$ \\
\hline \multicolumn{2}{|l|}{ Kidney disease } & $1.035(0.864$ to 1.240$)$ & 0.912 (0.758 to 1.098$)$ \\
\hline \multicolumn{2}{|l|}{ Cardiovascular } & $0.524(0.469$ to 0.584$)$ & 0.527 (0.472 to 0.588$)$ \\
\hline \multicolumn{4}{|l|}{ Low risk } \\
\hline Number of observations & 44011 & 44011 & 44011 \\
\hline Hosmer-Lemeshow goodness-of-fit & Pass & Pass & Pass \\
\hline C-statistic & 0.74 & 0.58 & 0.65 \\
\hline
\end{tabular}

Bold text indicates statistical significance at the $\alpha=0.05$ level on a two-tailed test estimated with stepdown Bonferroni correction of $p$ values. 

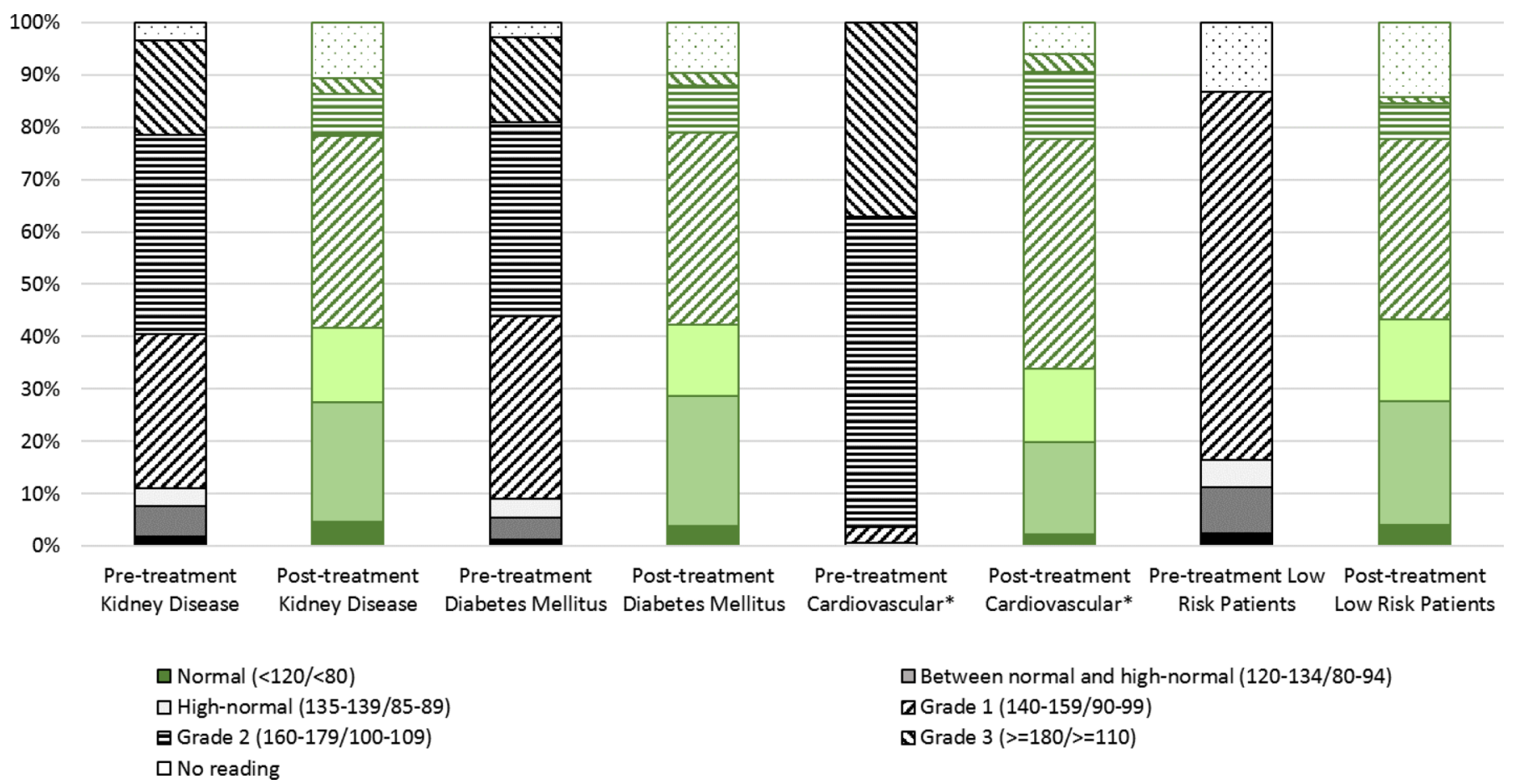

$\square$ Between normal and high-normal (120-134/80-94) 口Grade 1 (140-159/90-99)

DGrade $3(>=180 />=110)$

Figure 1 Percentage of patients in each blood pressure control group, pretreatment and post-treatment initiation, by risk cohort. *The cardiovascular risk group includes patients with grades 2 or 3 hypertension (with or without comorbid cardiovascular disease) prior to treatment initiation and those with 'high normal' or grade 1 hypertension plus one or more cardiovascular conditions (ie, coronary heart disease, cerebrovascular disease, peripheral vascular disease, myocardial infarction or hyperlipidaemia).

but generally positive, with $70.2 \%$ classified with grade 1 hypertension before treatment vs $42.5 \%$ with grade 1 or higher afterwards (figure 1). Overall, the proportion of patients who had BP readings at or below the "high normal' range increased more than sixfold following treatment initiation, from $5.8 \%$ to $37.4 \%$.

To better understand factors affecting treatment success, we modelled the likelihood of achieving BP control following treatment initiation as a function of therapeutic regimen (monotherapy vs combination therapy) controlling for demographics, comorbid conditions and lifestyle variables for all patients plus model variants run separately for low-risk and high-risk cohorts (table 4). Being older significantly decreases the odds of achieving BP goal for all patients except those with kidney disease or diabetes. Men with high cardiovascular risk are less likely than women in this group to achieve BP control. Having ever had a prior episode of hypertension treated in the past significantly reduced the odds of achieving control across all patient groups except those with kidney disease. Patients with diabetes (OR: 1.16; 95\% CI: 1.09 to 1.24) and kidney disease (OR: 1.18; 95\% CI: 1.09 to 1.28) were each slightly more likely to achieve BP control than other patients.

Current smokers with cardiovascular health conditions were less likely to reach BP target (OR: 0.87; $95 \%$ CI: 0.82 to 0.92$)$. Obesity reduced the odds of achieving goal for both cardiovascular risk patients (OR: 0.87; 95\% CI: 0.82 to 0.93 ) and those deemed low risk (OR: $0.86 ; 95 \% \mathrm{CI}$ : 0.77 to 0.95$)$. However, being merely overweight was associated with slightly higher odds of reaching goal among those in the cardiovascular high-risk group (OR: 1.11; $95 \%$ CI: 1.05 to 1.18 ).

Across all patients and risk subgroups, the odds of achieving BP control fell with increasing hypertension grade. For the full sample of patients, we found that starting on combination therapy increased the odds of achieving BP control relative to starting with monotherapy.

We performed a sensitivity analysis to assess the potential impact of missing data on BP readings by rerunning the analyses on all patients, first assuming that all patients with missing BP readings had achieved BP control following treatment and, second, assuming that they had not. There were no substantive changes in the coefficients under either scenario, although a few of the covariates (prior hypertension, current smoking status and overweight BMI) became insignificant under the first scenario assuming that all patients with missing BP recordings had met the BP control goal.

\section{DISCUSSION}

In line with the UK guidelines, we found that the majority of patients were initiated on single drug therapy. Few were treated with combination therapy and patients with diabetes or cardiovascular disease were less likely to receive combination drug treatment than patients with no risk factors. Treatment initiation was beneficial $(66.8 \%$ of patients had grade 2 or 3 hypertension pretreatment 


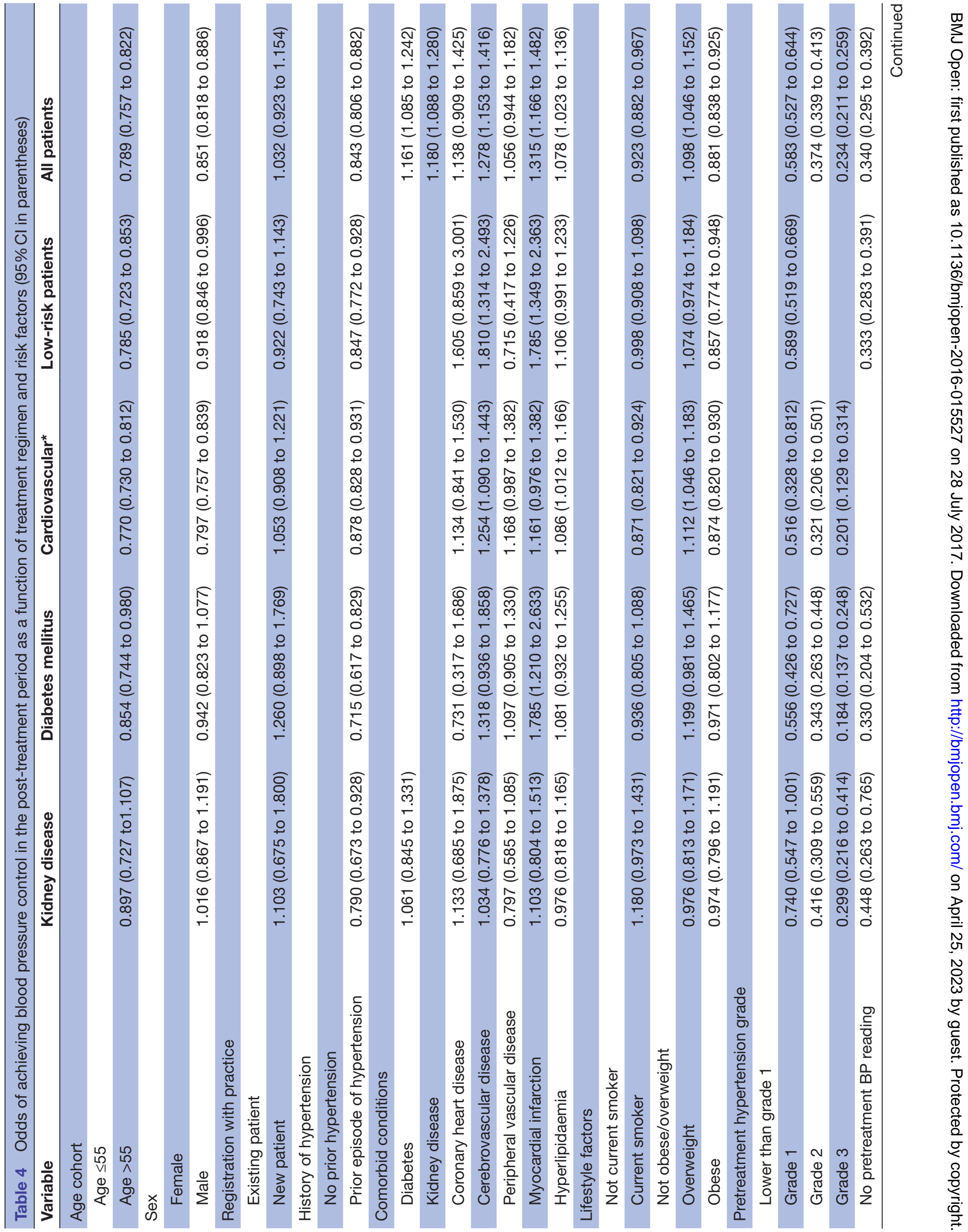




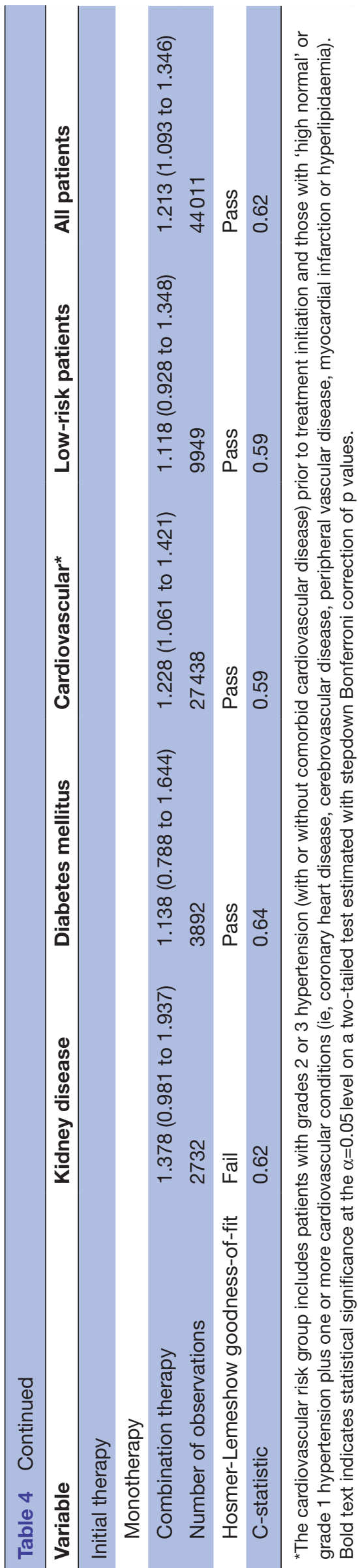

vs $13.5 \%$ post-treatment, overall). Patients with diabetes and kidney diseases were more likely than others to reach target BP readings. In addition, starting on combination therapy increased the odds of achieving BP control compared with starting on monotherapy.

\section{A population at risk}

This study of patients with newly treated hypertension in the UK found that a majority had risk factors complicating hypertension, including comorbid cardiovascular conditions, diabetes and/or kidney disease alone or in combination with index BP readings consistent with grade 2 or 3 hypertension. Many were also current smokers and/or were overweight or obese.

Conservative versus aggressive therapy for high-risk patients Our results may suggest that combination therapy is indicated for patients with grade 2 or 3 hypertension or high normal/grade 1 hypertension plus at least one cardiovascular condition. Although it is commonly thought that combination therapy is also necessary to attain BP control in patients with diabetes or kidney disease, our results showed that it was not a statistically significant predictor of reaching BP goals in these subgroups. Based on our findings, $60.6 \%$ of patients in our study population might have benefited more from initiation on multiple drugs. However, given that past NICE guidelines promulgate initiation on monotherapy, it is perhaps not surprising that we found that only $4.2 \%$ of patients started on combination therapy. The patients who may benefit most (eg, those in our cardiovascular high risk group) were actually the least likely to be prescribed combination therapy $(3.2 \%$ compared with $6.3 \%$ of patients with no risk factors), either in the form of fixed-dose combination pills or multidrug class prescriptions.

Following the recently published SPRINT study (a randomised trial of intensive vs standard BP control among patients with cardiovascular risk factors),${ }^{22}$ which was halted early owing to the finding that patients in the intensive arm (with goal systolic BP $<120 \mathrm{~mm} \mathrm{Hg}$ ) had lower rates of major cardiac events and lower rates of all-cause mortality than patients in the standard arm, it is likely that target BP readings for patients with cardiovascular risk factors will be lowered in the future. If so, more aggressive initial therapy for this risk cohort may be recommended.

\section{New BP goals and recommended therapies for patients with} diabetes or kidney disease

Controlling BP for subgroups of patients with diabetes and/or chronic kidney disease is particularly important as the combination of hypertension with either condition is associated with greatly increased risk of morbidity. ${ }^{11}$ Our multivariable analysis showed that patients with diabetes or chronic kidney disease were slightly more likely than other patients to meet the BP target of $<140 / 90$. Although at the time that our data were collected, the UK patients with these conditions had been encouraged to aim for even lower readings, more recent data suggest that meeting the general threshold may be preferable, 
prompting changes in both the European and the UK guidelines.

It has been found that reduction of systolic BP below $130 \mathrm{~mm} \mathrm{Hg}$ is quite difficult to achieve for patients with diabetes, ${ }^{11}$ and a reappraisal of the European guidelines undertaken in 2009, and the most recent European Society of Hypertension / European Society of Cardiology (ESHESC) guideline, backed off from recommendations of lower systolic BP goals for patients with diabetes and renal disease owing to a lack of clinical trial evidence of benefit from attaining the lower thresholds in these special populations. ${ }^{1123}$

Recently adopted NICE guidelines specific to patients with diabetes, set target BP at or below 140/90 unless there is kidney, eye or cardiovascular damage, in which case the goal is to keep BP $<130 / 80 \mathrm{~mm} \mathrm{Hg}$. Caution is urged in treating patients with diabetes too aggressively since the risk of adverse side effects, such as orthostatic hypotension, associated with use of antihypertensive medications is raised in patients with autonomic neuropathy. ${ }^{24}$ Some drug classes are not recommended owing to microvascular complications or metabolic problems. In general, ACEIs or ARBs are preferred as initial therapy, ${ }^{25}$ and we found that together these drugs as monotherapy accounted for $65 \%$ of index treatment regimens chosen for patients with diabetes. Although patients with diabetes had the lowest percentage use of diuretics of all risk groups, this drug class still accounted for $9.1 \%$ of initial therapy.

For patients with chronic kidney disease, BP targets do not differ from other patients. However, when the albumin/creatinine ratio (ACR) $\geq 30 \mathrm{mg} / \mathrm{mmol}$, ACEIs or ARBs are the recommended therapy. Other treatment pathways may be selected in the presence of hypertension with ACR $<30 \mathrm{mg} / \mathrm{mmol} .{ }^{26}$ We were not able to evaluate ACR levels. However, we did find that ACEs and ARBs accounted for $43.7 \%$ of index treatments offered to patients with kidney disease.

\section{Strengths and limitations of the study design and data}

This study was based on observations of a large, population-based sample of patients in real-world practice conditions. Retrospective analyses based on medical records that were collected for administrative purposes rather than for research are subject to limitations inherent in the data, including potentially incomplete reporting of certain data elements. One key study limitation is that our study population was identified in part using Read codes in the primary care setting only. Some patients with primary hypertension may have been missed or misclassified if Read codes were incorrectly recorded or missing. Evidence is lacking to validate the use of Read codes (vs repeated BP measurements) to identify cases of primary hypertension accurately.

Lack of complete data from inpatient and other encounter types may also have limited our ability to identify high-risk patients. Given that the UK GPs act as gatekeepers for specialist and non-emergency inpatient care, data are missing far less frequently than in other health data systems in the USA and Europe. Nevertheless, the prevalence of chronic conditions may be underestimated since diagnoses are not recorded at every visit. One UK study estimated that more than $25 \%$ of myocardial infarction events may be missed using primary care encounters data alone. ${ }^{27}$ We attempted to mitigate this problem by counting all recorded diagnoses available for each patient, including conditions reported prior to the start of the study period.

It was not possible to assess medication compliance in our population, since prescription data in medical records indicate the physician's intention, but do not directly reveal any information regarding patient compliance with prescribed therapies including whether prescriptions were filled. While it was possible to observe changes in prescribed medications, complete information was not available on the reasons for adding or changing medications (ie, owing to adverse effects or lack of effectiveness). A longer follow-up period would be needed to examine the impact of changes in drug therapy (eg, drug class, dose, fixed-drug or free-drug combinations) on BP control. A longer follow-up period would also be required to assess the long-term effect on BP outcomes of initial therapy choice.

Finally, selection bias may have been introduced in the regression analyses because of the necessity of limiting analysis of BP control to patients who had a follow-up BP readings recorded. Missing follow-up data on this key variable cannot be assumed to occur at random and may differ by risk cohort. However, the results of our sensitivity analysis suggest that the impact was minimal.

Guidelines have recently been updated and it would be interesting to assess whether this has had an impact on how newly diagnosed patients with hypertension are treated. While the NICE guidelines remain conservative, favouring monotherapy initially, key updates included the recommendation to offer antihypertensive drug therapy to patients with stage 2 hypertension, regardless of age, to patients with diabetes or renal disease, and to patients with 10 -year cardiovascular risk $\geq 20 \% .{ }^{10}$ Replicating these analyses for the period 2012 onwards to assess potential changes in practice patterns under the more recent NICE $^{102526}$ and European ${ }^{11}$ guidelines is warranted.

\section{CONCLUSION}

We report mixed findings on the adherence of physicians to best practice guidelines for special populations of high-risk patients in the UK. The NICE guideline CG34 of 2006-in effect during the study period-recommended to start conservatively with single drug class therapy for most patients and this seems to have been followed even in cases where a more aggressive approach might have been considered. One issue this study raised is that most patients treated for hypertension in the UK general practice are in fact high-risk patients. Patients with diabetes, for whom there are benefits to deferring 
a move to multidrug therapy, were found to be less likely than patients with no risk factors to be treated aggressively initially. However, patients with extremely high BP readings (grade 2 or 3 ) were also less likely than those with lower than grade 1 hypertension readings and no other risk factors to receive aggressive early therapy. The message that treatment must be tailored to the patient's individual risk profile needs greater emphasis, and this may mean backing away from the historically conservative approach taken by NICE except in the case of patients with lower grade hypertension and no other risk factors (see online supplementary file 1 ).

Contributors SW, AJ, JP and TT contributed to the conception or design of the work. SW was involved in data acquisition and carried out data analysis and interpretation. SW, AJ, JP and TT participated in the drafting of the article. SW and TT were responsible for the critical revision of the article. SW, AJ, JP and TT gave final approval of the version to be published.

Competing interests AJ and JP were employed by Takeda Europe, who provided funding for this study to PHMR. SW received consulting fees from PHMR to conduct the analyses and prepare the manuscript but has no ongoing conflict of interest in relation to the research results. TST has no personal, financial or institutional conflicts of interest associated with this work, nor has he ever received any fees or monetary compensation of any kind for his authorship contribution.

Ethics approval Scientific Review Committee, Cegedim Strategic Data Medical Research UK (now IMS Health).

Provenance and peer review Not commissioned; externally peer reviewed.

Data sharing statement № additional unpublished data from the study are available.

Open Access This is an Open Access article distributed in accordance with the Creative Commons Attribution Non Commercial (CC BY-NC 4.0) license, which permits others to distribute, remix, adapt, build upon this work non-commercially, and license their derivative works on different terms, provided the original work is properly cited and the use is non-commercial. See: http://creativecommons.org/ licenses/by-nc/4.0/

(c) Article author(s) (or their employer(s) unless otherwise stated in the text of the article) 2017. All rights reserved. No commercial use is permitted unless otherwise expressly granted.

\section{REFERENCES}

1. Kearney PM, Whelton M, Reynolds K, et al. Global burden of hypertension: analysis of worldwide data. Lancet 2005;365:217-23.

2. Forbes C, Lang S, Harker J, et al. The epidemiology of hypertension: a literature and systematic review. York, UK: Kleijnen Systematic Reviews Ltd, 2010.

3. World Health Organization (WHO). World Health Statistics 2012. Geneva, Switzerland: WHO Press, 2012. http://www.who.int/ gho/publications/world_health_statistics/EN_WHS2012_Full.pdf (accessed 9 Dec 2016).

4. Long AN, Dagogo-Jack S. Comorbidities of diabetes and hypertension: mechanisms and approach to target organ protection. $J$ Clin Hypertens 2011;13:244-51.

5. Murray CJ, Lopez AD. Evidence-based health policy--lessons from the global burden of disease study. Science 1996;274:740-3.

6. Ovbiagele B, Nguyen-Huynh MN. Stroke epidemiology: advancing our understanding of disease mechanism and therapy. Neurotherapeutics 2011;8:319-29.
7. Bui AL, Horwich TB, Fonarow GC. Epidemiology and risk profile of heart failure. Nat Rev Cardiol 2011;8:30-41.

8. Williams B, Poulter NR, Brown MJ, et al. British hypertension Society guidelines for hypertension management 2004 (BHS-IV): summary. BMJ 2004;328:634-40.

9. National Institute for Health and Clinical Excellence: CG34: Hypertension: NICE clinical guidelines. 1-6-2006London, UK National Institute for Health and Clinical Excellence $2006 \mathrm{http}: / /$ www.salforddiabetescare.co.uk/admin/resources/uploaded/NICE\% 20guidance\%20on\%20hypertension208.pdf (accessed 15 Apr 2017).

10. National Institute for Health and Clinical Excellence. CG127: Hypertension:Clinical management of primary hypertension in adults. 24-8-2011. London, UK: National Institute for Health and Clinical Excellence, 2011. https://www.nice.org.uk/guidance/cg127 (accessed 15 Apr 2017).

11. Mancia G, Fagard R, Narkiewicz K, et al. ESH/ESC guidelines for the management of arterial hypertension. Eur Heart J 2013;31:1281-357.

12. Health IMS. Data: statistics. 2015. http://www.epic-uk.org/our-data/ statistics.shtml (accessed 9 Dec 2016).

13. Health IMS. Cegedim Strategic Data: Data Content. 2015. http:// www.epic-uk.org/our-data/data-content.shtml (accessed 9 Dec 2016).

14. Lewis JD, Schinnar R, Bilker WB, et al. Validation studies of the health improvement network (THIN) database for pharmacoepidemiology research. Pharmacoepidemiol Drug Saf 2007;16:393-401.

15. Serumaga B, Ross-Degnan D, Avery AJ, et al. Effect of pay for performance on the management and outcomes of hypertension in the United Kingdom: interrupted time series study. BMJ 2011;342:d108.

16. MacDonald TM, Morant SV. Prevalence and treatment of isolated and concurrent hypertension and hypercholesterolaemia in the United Kingdom. Br J Clin Pharmacol 2008;65:775-86.

17. Harrison WN, Lancashire RJ, Marshall TP. Variation in recorded blood pressure terminal digit Bias in general practice. J Hum Hypertens 2008;22:163-7.

18. Health IMS. Cegedim Strategic Data: ethics. 2015. http://www.epicuk.org/our-data/ethics.shtml (accessed 9 Dec 2016).

19. von Elm E, Altman DG, Egger M, et al. The strengthening the Reporting of Observational studies in Epidemiology (STROBE) statement: guidelines for reporting observational studies. J Clin Epidemiol 2008;61:344-9.

20. National Health Service, Health and Social Care Information Centre. Quality and outcomes Framework Achievement Data 2008/09. 2013. http://content.digital.nhs.uk/catalogue/PUB04033/qof-eng-08-09bull-rep.pdf (accessed 17 Apr 2017).

21. Kramer AA, Zimmerman JE. Assessing the calibration of mortality benchmarks in critical care: the Hosmer-Lemeshow test revisited. Crit Care Med 2007;35:2052-6.

22. Wright JT, Williamson JD, Whelton PK, et al. A randomized trial of intensive versus standard blood-pressure control. N Engl J Med 2015;373:2103-16.

23. Mancia G, Laurent S, Agabiti-Rosei E, et al. European Society of Hypertension. Reappraisal of European guidelines on hypertension management: a European Society of Hypertension Task Force document. J Hypertens 2009;27:2121-58.

24. Naschitz JE, Slobodin G, Elias N, et al. The patient with supine hypertension and orthostatic hypotension: a clinical dilemma. Postgrad Med J 2006;82:246-53.

25. National Institute for Health and Clinical Excellence. NICE Pathways: Managing Blood Pressure in Type 2 Diabetes. London, UK: National Institute for Health and Care Excellence, 2013. https://www.nice.org. uk/guidance/ng28 (accessed 15 Apr 2017).

26. National Institute for Health and Clinical Excellence. NICE Pathways: possible complications of chronic kidney disease. London, UK: National Institute for Health and Care Excellence, 2013. https://www. nice.org.uk/guidance/cg182 (accessed 15 Apr 2017).

27. Herrett E, Shah AD, Boggon R, et al. Completeness and diagnostic validity of recording acute myocardial infarction events in primary care, hospital care, disease registry, and national mortality records: cohort study. BMJ 2013;346:f2350. 